Editorial

Z. Epileptol. 2021 ·34:129-131

https://doi.org/10.1007/s10309-021-00411-6

Angenommen: 8. März 2021

(c) Springer Medizin Verlag GmbH, ein Teil von Springer Nature 2021

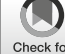

Adam Strzelczyk ${ }^{1}$. Friedhelm C. Schmitt ${ }^{2}$

'Epilepsiezentrum Frankfurt Rhein-Main, Zentrum der Neurologie und Neurochirurgie, GoetheUniversität Frankfurt, Frankfurt am Main, Deutschland

${ }^{2}$ Universitätsklinik für Neurologie, Otto-von-Guericke-Universität, Magdeburg, Deutschland

\title{
Syndromspezifische Therapien, Impfung gegen Corona und Epileptologie in der DDR
}

mission „Epilepsie und Synkopen“ der Deutschen Gesellschaft für Neurologie zur „Impfung zur Vorbeugung der COVID-19 Erkrankung sowie Impfpriorisierung bei Epilepsie“ [11]. Diese Publikation ist bereits am 01.02.2021 online erschienen und hatte das Ziel, Mitglieder der DGfE, Fachpublikum sowie Betroffene schnell und umfassend über epilepsiespezifische Fragen bezüglich der Impfung zu informieren. Bereits im ersten Monat nach der Online-Veröffentlichung ist diese Übersicht mehr als 1500-mal abgerufen worden. Erfreulicherweise wird für keinen der bereits zugelassenen Impfstoffe die Epilepsie als Kontraindikation ausgewiesen, ebenfalls werden epileptische Anfälle nicht als besondere Nebenwirkung der Impfung berichtet. In dem Sicherheitsbericht des Paul-Ehrlich-Instituts zu Verdachtsfällen von Nebenwirkungen und Impfkomplikationen nach Impfung zum Schutz vor COVID-19 werden zwar 51 Fälle epileptischer Anfälle in zeitlichem $\mathrm{Zu}$ sammenhang zur Verabreichung der drei bisher eingesetzten Impfstoffe genannt, jedoch überstieg die Anzahl dieser unerwünschten Ereignisse nicht die Anzahl der aufgrund der Hintergrundinzidenz in der Bevölkerung erwarteten Fälle, d.h. die Zahl der Meldungen lässt sich durch ein zeitlich zufälliges Zusammentreffen zwischen Impfung und Erkrankung interpretieren [12]. Seit der Publikation sind bereits weitere Änderungen der Impfpriorisierung in Deutschland erfolgt, insbesondere wurde die „Verordnung zum Anspruch auf Schutzimpfung gegen das Coronavirus SARS-CoV-2“
(Coronavirus-Impfverordnung - CoronaImpf V) im Bundesanzeiger am 08.02.2021 veröffentlicht [13], weitere Anpassungen werden in den nächsten Wochen sicherlich erfolgen. Insgesamt geben die neuen Empfehlungen den Behandelnden die Möglichkeit, Patienten mit chronischen Erkrankungen und in Zusammenschau der Befunde höher zu priorisieren. In Zusammenhang mit Epilepsie sei v. a. auf Menschen mit Epilepsie und geistiger Behinderung hingewiesen, die in die hohe Priorisierungsgruppe 2 eingeordnet werden können. Die Coronavirus-Impfverordnung ermöglicht ausdrücklich, dass bis zu zwei enge Kontaktpersonen von vulnerablen Impfberechtigten der Priorisierungsstufe 2 auch mit hoher Priorisierung geimpft werden können [13]. Beispielhaft seien hier die Eltern oder Betreuer von Kindern, Jugendlichen und Erwachsenen mit einer epileptischen Encephalopathie (z.B. Dravet-Syndrom oder Lennox-GastautSyndrom) zu nennen, die mit kognitiven und körperlichen Behinderungen einhergeht. In der aktuellen Empfehlung der DGfE [11] werden praktische Hinweise bezüglich einer Impfreaktion mit ggf. passagerer Gabe von fiebersenkenden Mitteln, wie Paracetamol oder Ibuprofen, oder von Benzodiazepinen gegeben. $\mathrm{Zu}$ beachten ist, dass die typische grippeähnliche Impfreaktion bei Verwendung der mRNA-Impfstoffe Comirnaty und Moderna eher und stärker bei der zweiten Impfung und bei Verwendung des Vektor-Impfstoffes AstraZeneca eher bei der ersten Impfung zu erwarten ist. Zeitgleich zu den oben genannten spezifisch
Empfehlung der Deutschen Gesellschaft
für Epileptologie (DGfE) sowie der Kom- 
auf die COVID-19 eingehenden Empfehlungen erfolgte durch Dr. Mayer aus Kleinwachau, Dr. Krämer aus Zürich und Prof. Brandl aus Jena eine Überarbeitung und Aktualisierung der generellen „Impfempfehlungen bei Epilepsie“ der DGfE. Diese sind ebenfalls online über die DGfE abrufbar [14].

Ein weiterer Beitrag in diesem Heft, der zwar wenig die tägliche klinische Arbeit bestimmt, aber nichtsdestotrotz von hohem Interesse ist, war ebenfalls Anlass für dieses Editorial der Herausgeber: Sie finden einen Beitrag von Prof. Rabending aus Greifswald, Dr. Harzer aus Süderholz und Prof. Runge aus Anklam zur Epileptologie in der DDR [15]. Prof. Rabending und Prof. Runge sind nicht nur langjährige Mitglieder der DGfE, sondern inzwischen auch beide Ehrenmitglieder. Sie wurden von den Herausgebern gebeten, einen Übersichtsartikel über die Versorgung von Menschen mit Epilepsie, die Organisation und technische Ausstattung in der DDR zusammenzustellen. Die Betrachtung dieses Abschnitts der jüngeren deutschen Geschichte erfolgt aus der Sicht der damaligen Behandler in Leitungspositionen an der Universitätsklinik Greifswald. Bereits im Reviewprozess ist deutlich geworden, dass es mehr als eine Sicht auf die Vorwendezeit gibt und die damalige Versorgungssituation sowie die Behandlung von Menschen mit Epilepsie unterschiedlich betrachtet werden kann. Wir sind sehr dankbar, dass die drei Autoren die Aufgabe, diese Übersicht $\mathrm{zu}$ verfassen, auf sich genommen haben. Unter anderem haben sie mehrere Weggefährten und Kollegen befragt und Archive durchgeschaut, um diese Zusammenstellung zu ermöglichen. Wir denken, dass dies ein sehr wichtiger Beitrag aus medizinhistorischer Sicht ist, der auch Anlass zu Diskussionen geben und somit zu einer weiteren Beschäftigung mit diesem Thema anregen wird. Hoffentlich führen diese dann auch zu weiteren Veröffentlichungen in der Zeitschrift für Epileptologie, schließlich ist der in diesem Heft veröffentlichte Beitrag einer der wenigen Beiträge in Fachjournalen, der sich mit diesem Abschnitt der jüngeren deutschen Geschichte der Epileptologie bzw. Neurologie beschäftigt [16].
Das kommende Heft 3 im Spätsommer 2021 hat die Telemedizin und Wearables zum Thema und wird von Dr. Mues aus Bochum und Prof. Surges aus Bonn zusammengestellt. Das Leitthema des letzten Heftes des Jahres 2021 wird von den Gastherausgeberinnen Dr. Haberlandt aus Dornbirn und Prof. Pataraia aus Wien betreut (Leitthema: „Akut-symptomatische Anfälle“). Wie immer möchten wir die Leserinnen und Leser unserer Zeitschrift ermuntern, auch zu den beiden nächsten Themenheften Beiträge spontan einzureichen. Seit Anfang des Jahres ist Frau Dr. Saskia Rehse für die Zeitschrift für Epileptologie beim Springer-Verlag in Heidelberg zuständig, und wir freuen uns auf die weitere Zusammenarbeit.

Ihre

\section{A. Strzelczyk \\ F.C. Schmitt}

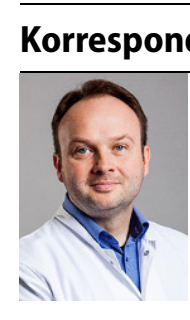

denzadresse

Prof. Adam Strzelczyk

Epilepsiezentrum Frankfurt

Rhein-Main, Zentrum

der Neurologie und

Neurochirurgie, Goethe-

Universität Frankfurt

Frankfurt am Main,

Deutschland

strzelczyk@

med.uni-frankfurt.de

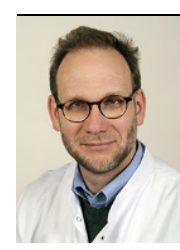

Dr. Friedhelm C. Schmitt

Universitätsklinik für

Neurologie, Otto-von-

Guericke-Universität

Magdeburg, Deutschland

fc.schmitt@t-online.de

Interessenkonflikt. A. Strzelczyk und F.C. Schmitt geben an, dass kein Interessenkonflikt besteht.

\section{Literatur}

1. French JA, Lawson JA, Yapici Z, Ikeda H, Polster $T$, Nabbout $R$ et al (2016) Adjunctive everolimus therapy for treatment-resistant focal-onset seizures associated with tuberous sclerosis (EXIST3): a phase 3, randomised, double-blind, placebocontrolled study. Lancet 388(10056):2153-2163. https://doi.org/10.1016/S0140-6736(16)31419-2

2. Willems LM, Strzelczyk A, Rosenow F (2021) Everolimus als krankheitsspezifische Therapieoption bei mit tuberöser Sklerose assoziierter, therapiere- fraktärer Epilepsie - ein systematischer Überblick. ZEpileptol 34(2). https://doi.org/10.1007/s10309020-00393-x

3. Schoonjans AS, Ceulemans B (2021) Dravet syndrome-toward an optimal and diseasespecific treatment. Z Epileptol 34(2). https://doi. org/10.1007/s10309-021-00399-z

4. Bast T (2021) Lennox-Gastaut-Syndrom: Wie behandeln? Z Epileptol 34(2). https://doi.org/10. 1007/s10309-021-00396-2

5. Nabbout R, Mistry A, Zuberi S, Villeneuve N, Gil-Nagel A, Sanchez-Carpintero R et al (2020) Fenfluramine for treatment-resistant seizures in patients with Dravet syndrome receiving Stiripentol-inclusive regimens: a randomized clinical trial. JAMA Neurol 77(3):300-308. https:// doi.org/10.1001/jamaneurol.2019.4113

6. Lagae L, Sullivan J, Knupp K, Laux L, Polster T, Nikanorova M et al (2019) Fenfluramine hydrochloride for the treatment of seizures in Dravet syndrome: a randomised, double-blind, placebo-controlled trial. Lancet 394(10216):2243-2254. https://doi. org/10.1016/S0140-6736(19)32500-0

7. Strzelczyk A, Schubert-Bast S (2020) Therapeutic advances in Dravet syndrome: a targeted literature review. Expert Rev Neurother 20(10):1065-1079. https://doi.org/10.1080/14737175.2020.1801423

8. Thiele EA, Bebin EM, Bhathal $H$, Jansen FE, Kotulska K, Lawson JA et al (2021) Add-on Cannabidiol treatment for drug-resistant seizures in tuberous sclerosis complex: a placebo-controlled randomized clinical trial. JAMA Neurol 78(3):285-292. https://doi.org/10.1001/jamaneurol.2020.4607

9. Wiemer-Kruel A (2021) Ketogene Ernährungstherapie unter besonderer Berücksichtigung der Behandlung der myoklonisch-astatischen Epilepsie. Z Epileptol 34(2). https://doi.org/10.1007/ s10309-020-00394-w

10. Klotz KA, Scheiwe C, Reinacher P, Antonio-Arce VS, Schulze-Bonhage A, Demerath T et al (2021) Kallosotomien beiSturzanfällen und epileptischen Spasmen. Z Epileptol 34(2). https://doi.org/10. 1007/s10309-021-00398-0

11. Strzelczyk A, Knake S, Holtkamp M, SchulzeBonhage $A$, Lemke J, von Spiczak $S$ et al (2021) Impfung zur Vorbeugung der COVID-19Erkrankung sowie Impfpriorisierung bei Epilepsie. ZEpileptol 34(2). https://doi.org/10.1007/s10309021-00404-5

12. Paul-Ehrlich-Institut (2021) Sicherheitsbericht: Verdachtsfälle von Nebenwirkungen und Impfkomplikationen nach Impfung zum Schutz vor COVID-19 seit Beginn der Impfkampagne am 27.12.2020 bis zum 26.02.2021. https://www. pei.de/SharedDocs/Downloads/DE/newsroom/ dossiers/sicherheitsberichte/sicherheitsbericht27-12-bis-26-02-21.pdf.Zugegriffen:23 Mär 2021

13. Bundesministerium für Gesundheit (2021) Verordnung zum Anspruch auf Schutzimpfung gegen das Coronavirus SARS-CoV-2 (Coronavirus-Impfverordnung - CoronalmpfV). https://www.bundesgesundheitsministerium.de/ fileadmin/Dateien/3_Downloads/C/Coronavirus/ Verordnungen/CoronalmpfV_BAnz_AT_08.02._ V1.pdf.Zugegriffen:4.März 2021

14. MayerT,KrämerG,BrandlU(2021)Impfempfehlungen derDGfE, Stand Januar 2021. http://www.dgfe. org/cweb2/cgi-bin-noauth/cache/VAL_BLOB/ 7872/7872/1923/ImpfempfehlungenderDGfEMärz-2021.pdf.Zugegriffen:24. Mär 2021

15. Rabending G, Harzer H, Runge U (2021) Epileptologie in der Deutschen Demokratischen Republik (DDR). Z Epileptol 34(2). https://doi.org/10.1007/ s10309-021-00408-1 
16. Teitge M, Kumbier E (2015) Zur Geschichte der DDR-Fachzeitschrift Psychiatrie, Neurologie und medizinische Psychologie (1949-1990). Nervenarzt 86(5):614-623. https://doi.org/10. 1007/s00115-014-4198-0

\section{Ekkehardt Kumbier (Hrsg.) \\ Psychiatrie in der DDR II}

Weitere Beiträge zur Geschichte

Berlin: be.bra wissenschaft verlag 2020, 432 S., (ISBN: 978-3-95410-263-1), 32 EUR

Als schwächster Punkt des Buches erscheint das Cover: Der Titel „Psychiatrie in der DDR II" ist weiß auf gelb gedruckt und schlecht zu lesen, darunter "Schriftenreihe zur MedizinGeschichte" noch schlechter, und der Name des Verlags lässt sich schließlich kaum noch entschlüsseln. Hinzu kommen zwei kontrastschwache gräuliche Reproduktionen von Fotos, die im Original höchstwahrscheinlich schwarz und scharf vorgelegen haben. Wer sich von diesem verunglückten Cover nicht abschrecken lässt, wird die Beiträge des Bandes (mit interessanten und scharfen Fotos bzw. Scans illustriert) mit umso größeren Genuss lesen. Sie gliedern sich in vier Kapitel: Gesellschaftlich-politischer Kontext, Fächerdifferenzierung und Spezialisierung, Diagnostische und therapeutische Ansätze und Entwicklungen in einzelnen psychiatrischen Kliniken.

Praktisch allen Beiträgen ist gemein, dass sie die DDR-Psychiatrie "sine ira et studio" untersuchen und diese in größere zeithistorische Kontexte einordnen. Die Autorlnnen meiden abstrakte Meta-Theorien und werten stattdessen eine Vielzahl von Archivalien aus. Hinzu kommen als Quelle etliche Zeitzeugen-Interviews, von denen klug Gebrauch gemacht wird, d.h. nicht als Ersatz, sondern als Ergänzung schriftlicher Dokumente. Ein Referat der 21 Beiträge ist hier schon aus Platzgründen nicht möglich, es sei dem Referenten deshalb erlaubt kurz vorzustellen, was inn bei der Lektüre besonders beeindruckte: Udo Grashoff berichtet über "Suizidprävention per Anruf: Staatliche Telefone des Vertrauens und kirchliche Telefonseelsorge in der DDR". Der Beitrag macht den umfassenden Regelungsanspruch der SED und das tiefe Misstrauen gegenüber privaten Initiativen, hier der Kirchen, sehr deutlich. Selbst der Vorsitzende des Ministerrates, Willi Stoph (1914-1999), musste sich mit diesem vergleichsweise "banalen“ Thema befassen! Hochpolitisch war hingegen "Der Pawlowismus in der frühen DDR", dem sich Steffen Dörre unter der Überschrift "Wissenschaft und Ideologie" widmet. Man kann nur staunen, wie sich von Freud geprägte Psychoanalytiker in den Dienst der neuen Lehre stellten, um ihr wenig später wieder abzuschwören. In diesen Kontext gehört auch "Die Karriere des Psychiaters Dietfried Müller-Hegemann", die Holger Steinberg als „Beispiel eines politisch gewollten Auf- und Abstiegs in der DDR" darstellt.

"Ich weiss, dass die Kur sehr hart wird..." beschreibt die Erfahrungen mit der DisulfiramAversionstherapie bei Alkoholikern, auch „Dialyseverfahren in der Therapie der Schizophrenien" (Ekkehardt Kumbier) werden behandelt - beides eine Warnung, Therapien modisch und unkritisch zu überhöhen.

Bemerkenswert erscheinen zudem die Beiträge, die sich mit literarischen oder filmischen Dokumenten zur Psychiatrie in der DDR befassen: Das bekannte Buch „Flucht in die Wolken", aber auch Gesundheitsfilme des Deutschen Hygiene-Museums Dresden und die aufsehenerregende Reportage "Die Hölle von Ueckermünde“ sind Gegenstand der Untersuchung.

Es bleiben zwei Wünsche: „Psychiatrie in der DDR III“ möge bald erscheinen - und ein Cover erhalten, das der Qualität des Inhalts entspricht.

Prof. Dr. Ulrich Meyer 